\title{
Cooperative Spectrum Detection Technology
}

\author{
Yujun Liu, Su Yan \\ Academy of Armored Forces Engineering \\ Beijing, 100072,China \\ yjliu@nudt.edu.cn
}

\begin{abstract}
This paper mainly discusses the cooperative spectrum sensing technologies in cognitive radio system. First, the paper starts with the single-node signal detection, then categorizes the spectrum sensing technologies and covers the good and weak points of the three major methods. Second, it puts forward a cooperative spectrum detection model and focuses on the effect of the cooperative detection and cooperative communication on the spectrum sensing performance. Last, with the help of mixed cooperative detection model, this paper compares the speed and accuracy of the single-node spectrum detection and that of the cooperative spectrum detection.
\end{abstract}

Keywords-Spectrum sensing, cooperative detection, dynamic distribution, spectrum correlation detection, interference temperature

\section{FOREWORD}

With the wide use of different radiofrequency equipment, the limited spectrum resources are under heavy pressure and the mutual interference between the equipment is increasing. At present, predominates the spectrum allocation method of fixed waveform parameters such as fixed central frequency, fixed modulation system, fixed frequency-hopping interval and fixed transmitter power, but this static spectrum management method can not solve the low efficiency of spectrum resource application in the complex electromagnetic environment and today's spectrum management method fails in the current electromagnetic environment.

The cognitive radio (CR) technology will fundamentally change the current radio communication system and spectrum management model. CR detects, analyzes, learn, evaluate and judge the surrounding electromagnetic environment, adjust its own state in real time and select the most suitable communication resource (such as frequency, modulation mode or transmitter power) so as to automatically help users choose the best radio transport channel for data transmission. So far, the environment cognitive ability poses the biggest challenge to the CR technology and it includes waveform cognition, spectrum cognition, network cognition, location cognition, available business cognition, user demand cognition, language cognition, state cognition, security strategy cognition, etc. The spectrum cognition calls for the quick and accurate positioning of the unoccupied frequency bands. If not, the inaccurate and delayed cognition results not only hinder the communication of the cognitive radio user but also harm the communication of licensed users. Therefore,

\author{
Ou Yang \\ Communication Engineering Dept. China Satellite Launch \\ and Tracking Control General, Beijing, 100120,China \\ ouy@cetin.net.cn
}

the speed and accuracy of spectrum cognition is one of the key technologies of cognitive radio.

\section{THE SPECTRUM SENSING OF CR}

The first step for $\mathrm{CR}$ to start communication is to detect spectrum holes and find the new ones suitable for communication. The ability of CR to detect and analyze the spectrum resources in a given area covers two aspects: first, the cognitive radio user has to detect the licensed user's signal of some frequency band to judge the status of the band. If it is vacant, the cognitive radio user can use it; if not, he has to look for another one. Second, when the cognitive radio user uses a certain frequency band, he has to continuously detect the environment, especially other vacant frequency bands. Once the licensed user appears, the cognitive will detect it in no time and switch into another frequency band as fast as possible or change its modulation mode so as not to delay the licensed user's communication.

According to the signals to be detected, the cognitive speed and accuracy usually fall into three categories:

First, when the licensed user signal structure is known to the cognitive radio user, the signal can be authenticated by the template-based matched filter. The good point of this method is that the cognitive radio user can accurately detect the appearance of the licensed user and take timely evasive action; however, the weak point is that when the channel quality is poor, the detection time is rather long and especially when the system is massive and has a large number of users, the cost of detecting equipment rockets.

Second, some parameters (such as center frequency and modulation mode) of the licensed user's signal are known to the cognitive radio user and then the signal can be checked with the relatively accurate signature detection which can further analyze the other features of the licensed user's signal for the signal has its own inherent features such as carrier frequency, bit rate and nested loop. The good point is that it has relatively high accuracy of detection while the weak one is that it needs a large amount of calculation and longer detection time than the first method.

Third, the licensed user's signal is totally strange to the cognitive radio user so he has to measure and analyze all the signals with a certain amount of energy, which means uncertain calculation task and analysis time. Therefore this method is less feasible.

As for the pure cognitive radio network, its spectrum cognition is much more difficult than that of the self-adaptive communication system. The major difficulties lie in not only the dynamic characteristics of wireless 
channels but also uncertainty of the network and sophistication of the equipment. For instance, the cognitive radio receiver must have higher sensitivity than the common user receiver. Any technology alone can not quickly and accurately conduct the spectrum detection. No matter what detection method is employed, the longer time the detection process requires, the more accurate it is. The quick spectrum detection is really a challenging task in actual communication.

\section{REALIZATION OF SPECTRUM SENSING}

Nowadays, the spectrum sensing technology focuses on detection of transmitters, interference-based detection and cooperative detection.

\section{A. Detection of transmitters}

From the perspective of traditional signal processing technology, the CR-based detection of signals of licensed users' transmitters consists in three means-matched filter detection, energy detection and static cyclical characteristic detection.

\section{1) Matched filter detection}

When the licensed user signal structure (such as modulation mode, waveform and format) is known to him, the cognitive radio user can quickly and accurately detect the licensed user's signal by using the matched filter. If nothing is known about the signal parameters, the detection result will be bad.

\section{2)Energy detection}

During the communication interval, the cognitive radio user can measure the energy of other signals in the main frequency band. If the energy is lower than the set threshold, the spectrum holes are believed to exist. The good point of energy detection is its simple operation while the weak point is that it can not distinguish modulation signals, noise and interference but just calculate the energy of signals, so the instantaneous signal to noise ratio affects the detection probability. The threshold of energy detection is likely to be affected by the noise power, so the detection of licensed user's pilot tone can be used together with the energy detection so as to reduce the interference.

\section{3)Static cyclic characteristic detection}

Modulation signals usually undergo treatments such as coding, carrier, frequency hopping spread spectrum and cyclic prefix, so they show a certain static period. The spectrum autocorrelation function helps detect their characteristics and distinguish between the signal energy and noise energy, so the method has high noise immunity. But the weak point lies in its sophisticated equipment and long detection time.

The transmitter-based detection performance is largely affected by the signal intensity especially in the environment where the multi-path fading and the shadowing effect are significant. Due to the measurement error and random change of thermal noise, interference is open to no accurate measurement. So a new method of interference measurement should be worked out which can be applied to both transmitters and receiving areas.

\section{B. Interference-based detection}

The Federal Communications Commission of the U.S. put forward the interference temperature model which focused the interference measurement on the self-adaptive real-time interactions between receivers and transmitters. The interference temperature model was defined as the sum of the radio frequency power of the unauthorized transmitter per unit bandwidth and the noise power of the receiver system and it took into full consideration the cumulative effect of all the interference. follows:

The interference temperature can be expressed as

$\mathrm{T} 1=(\mathrm{Ps}+\mathrm{P} 0) / \mathrm{KB}$, where $\mathrm{Ps}$ is referred to as the $\mathrm{RF}$ power of the unauthorized transmitter, $\mathrm{P} 0$ the noise power (unit: watt) of the receiver system, K Boerziman constant (1.38*10-23 joules/F) and B the signal bandwidth (unit: Hz). The interference temperature can be taken as the interference power spectral density in the frequency band. When the interference temperature estimation is lower than the set interference temperature threshold within given continuous time spans, the cognitive radio user can use the spectrum hole within this frequency band by adjusting its own parameters (such as transmitter power and modulation mode). The difficulty is how to accurately set the interference temperature threshold with no interference with the licensed user system. For example, when a licensed user has an exclusive use of one active channel, the CR node may not detect the signal and then will mistake it for an idle channel. So when the CR node increases its transmitter power, it may disrupt the licensed user's communication.

The TI Inc. proposed an improved method which employs a real-time database containing all information related to the current communication to replace the interference temperature measurement. Every node sends real-time transmitters' parameters to the database such as location, transmitter power and modulation mode. Contrary to the traditional way of measuring the signal-to-noise ratio at a presumed receiver position, CR will know which channel is available within its overage area by continuous monitoring of the database.

This method will fundamentally shift the research focus of the CR system which aims at creating an intelligent network instead of relying on the automatic intelligent equipment. The real-time database will contain all the necessary information which relates to the availability of unauthorized spectra, temporarily available spectra and spectra that can be rented for a short term and offers a real-time picture of all the spectra.

Though this method requires a lot of data to be processed, the huge benefit is that licensed users can exercise a complete control on their own spectra at any time. For example, when a licensed user wants to use the spectrum which is occupied by the cognitive radio user, the licensed user just needs to tell the database that this channel can not be used by others any more, and then he can start to use this channel in just a few seconds. This method will maximize the availability of spectra.

The above two methods are both based on the single cognitive radio user but neither the detection sensitivity nor 
the detection time is satisfactory. In this case, the multi-node spectrum cognition can surely boost the accuracy and stability of spectrum cognition.

\section{Cooperative detection}

Several cognitive radio users detect the licensed user's signal and exchange cognitive information. Then with the help of space analysis, the effect of multi-path fading is reduced. Meanwhile, the individual equipment is allowed lower sensitivity, which helps reduce sophistication of equipment.

Cooperative detection goes either in a centralized way or in a distributed way. The former way means that every cognitive radio user sends its own cognitive information to the data collection center (or the data fusion center) for data fusion and then the common final decision will be made by all the cognitive radio users involved in the detection; while the latter means the cognitive radio users exchange their cognitive information between themselves and then make their own decisions respectively.

\section{TO IMPROVE THE COOPERATIVE SPECTRUM COGNITION WITH THE HELP OF COOPERATIVE COMMUNICATION}

When cognitive radio users of a common cognitive radio system are using the idle channels unused by the licensed users, it is nothing but the use of spectra at different time by different users, which will not add to channel capacity and improve the transmission capability but raise the utilization rate of the spectra. The cooperative communication aims to boost the channel capacity but can do nothing about the idleness of the spectra. So the combination of the two technologies will help raise the anti-fading capability of the system and channel capacity as well as the utilization rate and the application efficiency of the spectra.

\section{A. Cooperative cognitive model}

At present, most cognitive radio systems are based on the multi-carrier modulation technique. Combining the multiple input and multiple output techniques with the current binding models, this paper comes up with three cooperative cognitive models which are based on multi-carrier modulation: cooperative spectrum detection, cooperative communication and the hybrid model. The cognitive radio user access is related to carrier distribution. Cognitive radio users and the licensed users can have their own communication respectively by competing for the use of the spectra at different time, which will raise the application efficiency of the spectra. They can also adopt cooperative communication techniques so as to raise both the application efficiency and the transmission capability. According to the users, the cooperation in the cognitive radio system falls into two categories: the cooperation between the cognitive radio users and the one between cognitive radio users and the licensed users.

1) Cooperative spectrum detection

Due to the fading effect, noise disturbance and such, cognitive radio users (CRU) may miss the spectrum detection or have wrong spectrum detection, which will cause serious disturbance to the licensed users (LU) or reduce the application efficiency of the spectra. There are two solutions to this problem. As for the first solution as illustrated in figure 1 (a), the licensed users will notify cognitive radio users of whether the spectra are used through broadcast. This solution is theoretically good and offers high accuracy of cooperative detection as well as sharp reduction of interference but it is necessary to change the licensed user communication protocol and install a special control channel between the licensed users and cognitive radio users. As for the second solution as illustrated in figure 1 (b), in some cases such as fading and when cognitive radio users are far away from the licensed users, if CRU2 can not accurately detect the idle spectra while CRU1 can, CRU1 and CRU2 will exchange spectrum detection information, which is sure to do CRU2 a favor. After the data from multi-node detection is fused, the detection accuracy improves. This method theoretically solves problems such as hidden terminals and the shadowing effect.

\section{2) Cooperative communication}

The precondition for cooperative communication is that the CRU has already detected the idle spectrum holes. The first solution is based on cognitive interference channels where the LU, as a source terminal, chooses one or more cognitive radio users as the repeater to transmit its information, which equals the multi-path transmission. This method improves the transmission efficiency, reduces the time when the LU occupies the spectrum and provides cognitive radio users with more chances for spectrum access. With the second solution, cognitive radio users work as the repeater for each other adopting semi-duplex or full-duplex mode. In semi-duplex mode, TDMA is used for spectrum access and the repeater (e.g. CRU2) will not transmit its own information but relay the source terminal's (e.g. CRU1) information while in full-duplex mode, CDMA is used and cognitive radio users work as the repeater for each other. In this case, cognitive radio users will transmit their own information and also relay other cognitive radio users' information.

In actual communication, cooperative detection will use the above two models. The hybrid model works as illustrated in figure 1: first, spectrum detection is carried out and then cooperative communication follows. During this process, different users can be chosen as partners.

\section{B. Mixed cooperative detection}

Based on the model illustrated in figure 1 and the Rayleish fading channel, an assumption is worked out: the spectrum detection probability of every cognitive radio user is bigger than 0.8 , cooperative communication adopts the equal power distribution algorism, a certain number of cognitive radio users are involved in cooperative spectrum detection, different signal-to-noise ratios are assigned to different users and a user is chosen at random as a partner in cooperative communication.

When the signal-to-noise ratio is high, the accuracy of cooperative detection is almost the same as that of single cognitive radio user detection. When the error-code quotiety 
is bigger than $10-3$ and $\mathrm{n}$ (the number of cooperative detection nodes) is no smaller than 3 , the accuracy of mixed cooperative detection is closer to the real picture of the spectra. However, in a network with limited resources, it is necessary to set the transmission efficiency as the weight and to properly weight the detection result of the cognitive radio users having better transmission capability so as to increase the detection accuracy. As a result, the detection probability of the idle licensed user spectra improves both in a fading environment and a non-fading one. In this case, the idle licensed user spectra will be better used.

In a CR network, every node serves as both a cognitive terminal and a data fusion center, which requires a special control channel to be installed to transmit detection data. When the data volume surges, communication overhead increases, which is liable to burden both the cognitive network resources and the traditional network ones. The key factors which affect the performance of the mixed cooperative detection include the cognitive capability of the individual user involved in the cooperative detection, the network topological capacity and data fusion techniques.

\section{CONCLUSION}

The IEEE and ITU have done a lot of work to set standards. The CR standards the IEEE are working on include 802.22, 802.16h, SCC41/ P1900, 802.11h and 802.11y and these standards turn CR from concept into reality. CR sheds a new light on how to solve the contradiction between the increasing demand for wireless communication and limited wireless spectrum resources and is likely to offer a new direction for the future wireless communication development and become a new approach to spectrum management. CR still faces many challenges as in its theory, design and engineering and quite needs tests of massive deployment and application. Nowadays, wireless spectrum resources are still confined to authorized distribution and this mode of spectrum distribution will remain unchanged in the foreseeable future but CR surely works to make it better.

\section{REFERENCES}

[1] Joseph Mitola. COGNITIVE RADIO ARCHITECTURE [M]. New Jersey U.S.: A JOHN WILEY \& SONS, INC., PUBLICATION, 2006.

[2] Bruce A. Fette. Cognitive radio technology—1st ed, [M]. Linacre House, Jordan Hill, Oxford OX2 8DP, UK, 2006.

[3] Daniel G. Mock. Software Radios for the Military [C]. Warsaw, Poland: RTO IST Symposium on "Military Communications", 2001.

[4] Ben Wild, Kannan Ramchandran: detecting primary receivers for cognitive radio[C], IEEE, 2005:124-130

[5] LEE J, KIM YOUNG Min. Weighted-cooperative spectrum sensing scheme using clustering in cognitive radio systems[C]. IEEE ICACT 2008, 2008:786-790

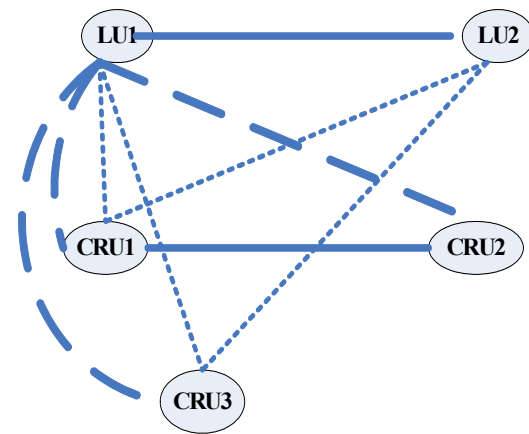

(a) cooperative spectrum detection and cooperative communication between LU and CRU

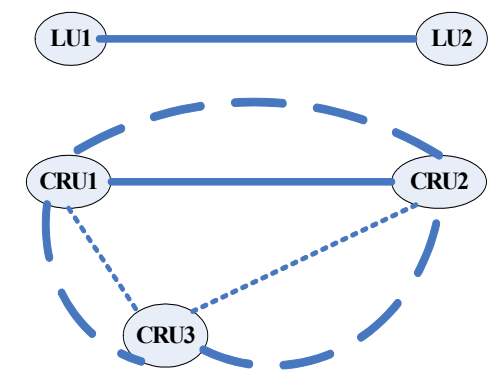

(b) cooperative spectrum detection and semiduplex cooperative communication between CRU

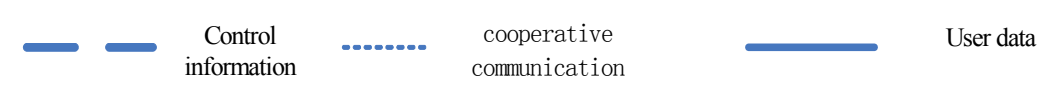

Figure 1. Cooperative detection data interaction 